\title{
Winding expansion techniques for lattice QCD with chemical potential
}

\author{
Julia Danzer and Christof Gattringer \\ Institut für Physik, FB Theoretische Physik, Universität Graz \\ 8010 Graz, Austria
}

(Dated: September 16, 2008)

\begin{abstract}
We analytically derive a decomposition of the lattice fermion determinant for Wilson's Dirac operator with chemical potential into winding sectors, i.e., factors with a fixed number of quarks. Dividing the lattice into four domains, the determinant is factorized into terms which can be classified with respect to the winding number of the closed loops they consist of. The individual factors are expressed in terms of subdeterminants and propagators on the domains of the lattice. We numerically analyze properties of the factorization formula and discuss two applications for the determination of canonical partition functions with a fixed quark number: A speedup for the Fourier transformation technique through a dimensional reduction, and a power series expansion.
\end{abstract}

PACS numbers: 11.15.Ha

\section{INTRODUCTION}

The lattice formulation provides a fully gauge invariant non-perturbative approach to QCD. An important aspect of the lattice discretization is the possibility of using numerical Monte Carlo techniques to obtain non-perturbative results. An application where the success so far was rather limited is lattice QCD with chemical potential. The reason is the sign problem of the fermion determinant with a chemical potential, which forbids a direct application of the Monte Carlo method such that one is reduced to reweighting methods.

In these attempts the use of canonical partition functions with a fixed quark (or baryon) number has started to play an important role recently [1][4]. For their use it is necessary to have an efficient method for evaluating the canonical partition function at a fixed quark number. This problem is in turn related to organizing the fermion determinant, which may be viewed as a collection of closed loops, with respect to the winding number of these loops around compactified time.

In this article we present an explicit decomposition of the fermion determinant for Wilson's Dirac operator into sectors with definite winding numbers. The approach is based on a partition of the lattice into four domains, and the contributions to the individual winding sectors may be written in terms of propagators on these lattice domains. The terms in in our factorization formula for the fermion determinant are analyzed numerically. We discuss two possible applications of our results to the evaluation of the canonical partition function at fixed quark number.

\section{PARTITION OF THE LATTICE INTO FOUR DOMAINS}

The starting point of our approach is a partition of a $L^{3} \times \beta$ lattice $\Lambda$ into four domains with different ranges of the time component $x_{4}$ :

$\Lambda^{(1)}=\left\{\left(\vec{x}, x_{4}\right) \mid \vec{x} \in \Lambda_{s}, x_{4}=-\beta / 2+1, \ldots-1\right\}$, $\Lambda^{(2)}=\left\{\left(\vec{x}, x_{4}\right) \mid \vec{x} \in \Lambda_{s}, x_{4}=0\right\}$, $\Lambda^{(3)}=\left\{\left(\vec{x}, x_{4}\right) \mid \vec{x} \in \Lambda_{s}, x_{4}=1, \ldots \beta / 2-1\right\}$, $\Lambda^{(4)}=\left\{\left(\vec{x}, x_{4}\right) \mid \vec{x} \in \Lambda_{s}, x_{4}=\beta / 2\right\}$.

We stress that $\beta$ is a positive integer, the inverse temperature in lattice units. $\Lambda_{s}$ denotes the spatial part of the lattice. The full lattice $\Lambda$ is the union of the four sub-lattices $\Lambda^{(i)}, i=1,2,3,4$. The partition of the lattice is illustrated in Fig. 1]

Wilson's lattice Dirac operator $D(x, y)$ which we use here can be decomposed into pieces $D^{(i)}$ acting in an individual domain $\Lambda^{(i)}$ and terms $D^{(i, j)}$ that connect the neighboring domains $\Lambda^{(i)}, \Lambda^{(j)}, i \neq j$. For that purpose we need the operators

$$
\begin{aligned}
D^{(i)}(x, y) & : x, y \in \Lambda^{(i)}, i=1,2,3,4, \\
D^{(1,2)}(x, y) & : \vec{x}, \vec{y} \in \Lambda_{s}, x_{4}=-1, y_{4}=0, \\
D^{(2,1)}(x, y) & : \vec{x}, \vec{y} \in \Lambda_{s}, x_{4}=0, y_{4}=-1, \\
D^{(2,3)}(x, y) & : \vec{x}, \vec{y} \in \Lambda_{s}, x_{4}=0, y_{4}=1, \\
D^{(3,2)}(x, y) & : \vec{x}, \vec{y} \in \Lambda_{s}, x_{4}=1, y_{4}=0, \\
D^{(3,4)}(x, y) & : \vec{x}, \vec{y} \in \Lambda_{s}, x_{4}=\beta / 2-1, y_{4}=\beta / 2, \\
D^{(4,3)}(x, y) & : \vec{x}, \vec{y} \in \Lambda_{s}, x_{4}=\beta / 2, y_{4}=\beta / 2-1, \\
D^{(4,1)}(x, y) & : \vec{x}, \vec{y} \in \Lambda_{s}, x_{4}=\beta / 2, y_{4}=-\beta / 2+1, \\
D^{(1,4)}(x, y) & : \vec{x}, \vec{y} \in \Lambda_{s}, x_{4}=-\beta / 2+1, y_{4}=\beta / 2,
\end{aligned}
$$

where we also display which values the arguments $x$ and $y$ may assume. The term $D^{(1)}$ is given by (we 


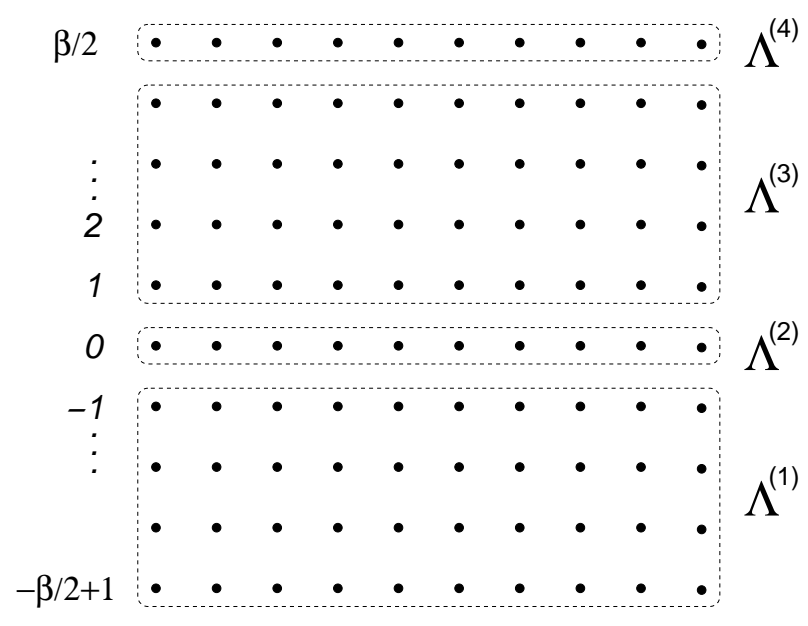

FIG. 1: Partition of the lattice into four domains $\Lambda^{(i)}, i=1,2,3,4$ : The vertical direction in the plot is time, while the dots in horizontal direction represent a whole time slice. On the left-hand side we indicate the values of the time argument. The lattice sites in the same domain are enclosed in a dashed contour, and we label the domains on the right-hand side.

set the lattice constant to 1 throughout this paper and all numerical results are given in lattice units)

$$
\begin{aligned}
& D^{(1)}(x, y)=\delta_{\vec{x}, \vec{y}} \delta_{x_{4}, y_{4}} \\
& -\kappa \sum_{j= \pm 1}^{ \pm 3} \frac{1 \mp \gamma_{|j|}}{2} U_{j}\left(\vec{x}, x_{4}\right) \delta_{\vec{x}+\hat{j}, \vec{y}} \delta_{x_{4}, y_{4}} \\
& -\kappa \sum_{n_{4}=-\frac{\beta}{2}+1}^{-2} \frac{1-\gamma_{4}}{2} U_{4}\left(\vec{x}, n_{4}\right) \delta_{\vec{x}, \vec{y}} \delta_{x_{4}, n_{4}} \delta_{y_{4}, n_{4}+1} \\
& -\kappa \sum_{n_{4}=-\frac{\beta}{2}+2}^{-1} \frac{1+\gamma_{4}}{2} U_{4}\left(\vec{x}, n_{4}-1\right)^{\dagger} \delta_{\vec{x}, \vec{y}} \delta_{x_{4}, n_{4}} \delta_{y_{4}, n_{4}-1}
\end{aligned}
$$

where we have defined the hopping parameter $\kappa=$ $1 /(4+m)$ with $m$ being the bare quark mass $[9]$. We use the convention $U_{-j}\left(\vec{x}, x_{4}\right)=U_{j}\left(\vec{x}-\hat{j}, x_{4}\right)^{\dagger}$, where $\hat{j}$ denotes the unit vector in $j$-direction. The term $D^{(3)}$ is obtained by shifting the range of the auxiliary sums running over $n_{4}$ in the last two terms of (3) by $\beta / 2$.

The terms $D^{(2)}$ and $D^{(4)}$ live on only a single time slice, with $D^{(2)}$ given by

$$
\begin{aligned}
& D^{(2)}(x, y)=\delta_{\vec{x}, \vec{y}} \delta_{x_{4}, 0} \delta_{y_{4}, 0} \\
& -\kappa \sum_{j= \pm 1}^{ \pm 3} \frac{1 \mp \gamma_{|j|}}{2} U_{j}\left(\vec{x}, x_{4}\right) \delta_{\vec{x}+\hat{j}, \vec{y}} \delta_{x_{4}, 0} \delta_{y_{4}, 0} .
\end{aligned}
$$

$D^{(4)}$ is obtained from $D^{(2)}$, by setting the time arguments in the temporal Kronecker deltas to $\beta / 2$.

The terms that connect the domains $\Lambda^{(i)}$ have only hops in time direction, with

$$
\begin{aligned}
& D^{(1,2)}(x, y)=-\kappa \frac{1-\gamma_{4}}{2} U_{4}(\vec{x},-1) \delta_{\vec{x}, \vec{y}} \delta_{x_{4},-1} \delta_{y_{4}, 0}, \\
& D^{(2,1)}(x, y)=-\kappa \frac{1+\gamma_{4}}{2} U_{4}(\vec{x},-1)^{\dagger} \delta_{\vec{x}, \vec{y}} \delta_{x_{4}, 0} \delta_{y_{4},-1},
\end{aligned}
$$

and the terms $D^{(2,3)}, D^{(3,2)}, D^{(3,4)}$ and $D^{(4,3)}$ are obtained from $D^{(1,2)}$ and $D^{(2,1)}$ by setting the time arguments according to the values listed in (2).

The terms $D^{(1,4)}$ and $D^{(4,1)}$, which periodically close the lattice in time direction, have a slightly different form, since here the chemical potential $\mu$ is coupled and the anti-periodic boundary conditions in time are implemented. We write the two terms as

$$
D^{(4,1)}=e^{\mu \beta} D_{0}^{(4,1)}, D^{(1,4)}=e^{-\mu \beta} D_{0}^{(1,4)},
$$

with the $\mu$-independent pieces

$$
\begin{aligned}
& D_{0}^{(4,1)}(x, y)=\kappa \frac{1-\gamma_{4}}{2} U_{4}(\vec{x}, \beta / 2) \delta_{\vec{x}, \vec{y}} \delta_{x_{4}, \frac{\beta}{2}} \delta_{y_{4},-\frac{\beta}{2}+1} \\
& D_{0}^{(1,4)}(x, y)=\kappa \frac{1+\gamma_{4}}{2} U_{4}(\vec{x}, \beta / 2)^{\dagger} \delta_{\vec{x}, \vec{y}} \delta_{x_{4},-\frac{\beta}{2}+1} \delta_{y_{4}, \frac{\beta}{2}}
\end{aligned}
$$

Combining all terms $D^{(i)}$ on the individual domains and the connecting pieces $D^{(i, j)}$, one obtains the standard Wilson Dirac operator with the chemical potential introduced as a boundary condition.

For later use we remark that the individual terms obey the following $\gamma_{5}$-hermiticity properties:

$$
\begin{aligned}
& D^{(i) \dagger}=\gamma_{5} D^{(i)} \gamma_{5}, i=1,2,3,4 \\
& D^{(i, j) \dagger}=\gamma_{5} D^{(j, i)} \gamma_{5}, \quad(i, j)=(1,2),(2,3),(3,4) \\
& D_{0}^{(4,1) \dagger}=\gamma_{5} D_{0}^{(1,4)} \gamma_{5} .
\end{aligned}
$$

We remark at this point, that our approach may be implemented in the same way for clover improved Wilson fermions and also staggered fermions. For the latter case a dimensional reduction formula obtained in a different way can be found in [1, [5].

\section{FACTORIZATION OF THE DETERMINANT}

We now use the partition of the lattice into domains to factorize the fermion determinant. The basic step is to write the Grassmann integral for the determinant in terms of the Grassmann fields orga- 
nized according to the domains $\Lambda^{(i)}$ :

$$
\begin{aligned}
\operatorname{det}[D]= & \int \prod_{i=1}^{4} d \bar{\psi}^{(i)} d \psi^{(i)} \exp \left(-\sum_{j=1}^{4} \bar{\psi}^{(j)} D^{(j)} \psi^{(j)}\right. \\
& -\bar{\psi}^{(1)} D^{(1,2)} \psi^{(2)}-\bar{\psi}^{(2)} D^{(2,1)} \psi^{(1)} \\
& -\bar{\psi}^{(2)} D^{(2,3)} \psi^{(3)}-\bar{\psi}^{(3)} D^{(3,2)} \psi^{(2)} \\
& -\bar{\psi}^{(3)} D^{(3,4)} \psi^{(4)}-\bar{\psi}^{(4)} D^{(4,3)} \psi^{(3)} \\
& \left.-\bar{\psi}^{(4)} D^{(4,1)} \psi^{(1)}-\bar{\psi}^{(1)} D^{(1,4)} \psi^{(4)}\right)
\end{aligned}
$$

Here we use vector/matrix notation for all indices, color, Dirac and space-time. The superscripts attached to the Grassmann variables $\bar{\psi}^{(i)}, \psi^{(i)}$ denote which domains of the lattice these spinors live on. The sum of all terms in the exponential function reproduces Wilson's fermion action with chemical potential.

We now first integrate out the Grassmann variables in the domains $\Lambda^{(1)}$ and $\Lambda^{(3)}$, then in domain $\Lambda^{(2)}$ and finally in $\Lambda^{(4)}$. Solving this chain of Gaussian integrals, one may write the determinant as

$$
\operatorname{det}[D]=A_{0} W
$$

with

$$
\begin{gathered}
A_{0}=\operatorname{det}\left[D^{(1)}\right] \operatorname{det}\left[D^{(3)}\right] \operatorname{det}\left[\widetilde{D}^{(2)}\right] \operatorname{det}\left[\widetilde{D}^{(4)}\right] \\
W=\operatorname{det}\left[1-\widetilde{S}^{(4)}\left[e^{\mu \beta} \widetilde{D}_{1}^{(4,2)}+\widetilde{D}_{3}^{(4,2)}\right]\right. \\
\left.\quad \times \widetilde{S}^{(2)}\left[e^{-\mu \beta} \widetilde{D}_{1}^{(2,4)}+\widetilde{D}_{3}^{(2,4)}\right]\right]
\end{gathered}
$$

We have introduced the following abbreviations:

$$
\begin{aligned}
& S^{(1)}=\left(D^{(1)}\right)^{-1}, S^{(3)}=\left(D^{(3)}\right)^{-1}, \\
& \widetilde{D}^{(2)}=D^{(2)}-D^{(2,1)} S^{(1)} D^{(1,2)}-D^{(2,3)} S^{(3)} D^{(3,2)} \\
& \widetilde{D}^{(4)}=D^{(4)}-D_{0}^{(4,1)} S^{(1)} D_{0}^{(1,4)}-D^{(4,3)} S^{(3)} D^{(3,4)} \\
& \widetilde{S}^{(2)}=\left(\widetilde{D}^{(2)}\right)^{-1}, \widetilde{S}^{(4)}=\left(\widetilde{D}^{(4)}\right)^{-1}, \\
& \widetilde{D}_{1}^{(4,2)}=D_{0}^{(4,1)} S^{(1)} D^{(1,2)} \\
& \widetilde{D}_{3}^{(4,2)}=D^{(4,3)} S^{(3)} D^{(3,2)} \\
& \widetilde{D}_{1}^{(2,4)}=D^{(2,1)} S^{(1)} D_{0}^{(1,4)} \\
& \widetilde{D}_{3}^{(2,4)}=D^{(2,3)} S^{(3)} D^{(3,4)} .
\end{aligned}
$$

The individual terms have an interesting hierarchical structure: $D^{(1)}$ and $D^{(3)}$ simply are the Dirac operators on the lattice domains $\Lambda^{(1)}$ and $\Lambda^{(3)}$. The corresponding propagators are denoted by $S^{(1)}$ and $S^{(3)}$.

To leading order the operator $\widetilde{D}^{(2)}$ (and similarly $\left.\widetilde{D}^{(4)}\right)$ is also the Dirac operator on the corresponding domain $\Lambda^{(2)}$ (and $\Lambda^{(4)}$ respectively). However, also two additional correction terms appear: In the first term the temporal links in $D^{(2,1)}$ allow a quark to hop into the neighboring domain $\Lambda^{(1)}$. There it may propagate with the propagator $S^{(1)}$, and in the end is again transported back into $\Lambda^{(2)}$ by the link terms in $D^{(1,2)}$. The second correction term is built in the same way, now allowing the quark to propagate in $\Lambda^{(3)}$. The term $\widetilde{D}^{(4)}$ has exactly the same structure: It adds to the Dirac operator in $\Lambda^{(4)}$ additional terms that allow a quark to visit the neighboring domains $\Lambda^{(1)}$ and $\Lambda^{(3)}$

A useful way of thinking about the individual contributions is in terms of paths: The propagators $S^{(1)}$ and $S^{(3)}$ may be viewed as collections of paths that connect any point in $\Lambda^{(1)}\left(\Lambda^{(3)}\right)$ with any other point. Along these paths ordered products of the gauge links $U_{\mu}(x)$ appear, such that the individual contributions are gauge covariant. The correction terms in $\widetilde{D}^{(2)}$ and $\widetilde{D}^{(4)}$ thus are paths in the neighboring domains $\Lambda^{(1)}$ and $\Lambda^{(3)}$ which are connected to the time slices $\Lambda^{(2)}$ and $\Lambda^{(4)}$ through temporal hops.

It is important to note, that the four determinants in the factor $A_{0}$ (11) do not depend on the chemical potential $\mu$. The only term where the chemical potential could appear in $A_{0}$ is the first correction term in $\widetilde{D}^{(4)}$. However, since there both $D^{(4,1)}$ and $D^{(1,4)}$ appear, the factors $\exp ( \pm \mu \beta)$ cancel, and only the $\mu$-independent terms $D_{0}^{(4,1)}$ and $D_{0}^{(1,4)}$ remain. As a consequence one expects that the four determinants are real. For $\operatorname{det}\left[D^{(1)}\right]$ and $\operatorname{det}\left[D^{(3)}\right]$ this follows from the $\gamma_{5}$-hermiticity of $D^{(1)}$ and $D^{(3)}$ (see Eq. (8)). Also the corrected Dirac operators $\widetilde{D}^{(2)}$ and $\widetilde{D}^{(4)}$ are $\gamma_{5}$-hermitian, as can be established with the help of the relations in (8). The corresponding determinants thus also are real.

We conclude, that the complex phase must be contained in the factor $W$ defined in (11), where we explicitly see terms that do depend on the chemical potential $\mu$. In the factor $W$ new types of matrices appear, such as the matrix $\widetilde{D}_{1}^{(4,2)}$ : This matrix connects the domains $\Lambda^{(4)}$ and $\Lambda^{(2)}$ by first hopping from $\Lambda^{(4)}$ into $\Lambda^{(1)}$ with the temporal hops in $D_{0}^{(4,1)}$, subsequently transporting a quark with $S^{(1)}$, and finally connecting to $\Lambda^{(2)}$ with the hops in $D^{(1,2)}$. Thus $\widetilde{D}_{1}^{(4,2)}$ may be considered as a large forward hopping term from $\Lambda^{(4)}$ into $\Lambda^{(2)}$ going through $\Lambda^{(1)}$. In the same way $\widetilde{D}_{3}^{(4,2)}$ can be viewed as a large backward hopping term from $\Lambda^{(4)}$ into $\Lambda^{(2)}$ going through $\Lambda^{(3)}$. The other two new terms $\widetilde{D}_{3}^{(2,4)}$ and $\widetilde{D}_{1}^{(2,4)}$ have a similar interpretation as large forward and backward hopping terms.

We stress at this point, that the paths which the large hops consist of have a length of $\beta / 2$ or longer, i.e., they consist of at least $\beta / 2$ individual steps. Let us discuss this property for the example of $D_{1}^{(4,2)}$ : According to (12) this term is given by the the product $D_{0}^{(4,1)} S^{(1)} D^{(1,2)}$. The first and the last factor each contribute one temporal step. The propagator 
$S^{(1)}$ is a collection of arbitrary paths, but here we only need to consider the paths that extend from the timeslice with $x_{4}=-\beta / 2+1$ to the timeslice at $x_{4}=-1$, since otherwise the terms $D_{0}^{(4,1)}$ and $D^{(1,2)}$ could not attach to $S^{(1)}$ (compare Fig. 1). The paths that connect the two boundaries of $\Lambda^{(1)}$ have at least a length of $\beta / 2-2$ steps, which establishes, that $D_{1}^{(4,2)}$ indeed consists of paths which have at least the length $\beta / 2$, and the same holds for $D_{3}^{(4,2)}, D_{1}^{(2,4)}$ and $D_{3}^{(2,4)}$.

Finally we remark that also the large hopping terms are related to each other through $\gamma_{5^{-}}$ hermiticity:

$$
\begin{aligned}
& D_{1}^{(2,4) \dagger}=\gamma_{5} D_{1}^{(4,2)} \gamma_{5}, \\
& D_{3}^{(2,4) \dagger}=\gamma_{5} D_{3}^{(4,2)} \gamma_{5} .
\end{aligned}
$$

Effectively the factor $W$ has the structure of a determinant with only two timeslices given by the domains $\Lambda^{(2)}$ and $\Lambda^{(4)}$. With the large hops $\widetilde{D}_{1}^{(2,4)}$, $\widetilde{D}_{3}^{(2,4)}, \widetilde{D}_{1}^{(4,2)}$ and $\widetilde{D}_{3}^{(4,2)}$ we can hop between these timeslices. The terms $S^{(4)}$ and $S^{(2)}$ that appear in $W$ between the large hops allow for a local propagation within $\Lambda^{(2)}$ and $\Lambda^{(4)}$.

\section{DECOMPOSITION INTO WINDING SECTORS}

The factor $W$ of (11) may be expanded with the trace-log formula for determinants. For that purpose we introduce the abbreviations

$$
\begin{aligned}
& H_{0}=\widetilde{S}^{(4)} D_{1}^{(4,2)} \widetilde{S}^{(2)} D_{1}^{(2,4)}+\widetilde{S}^{(4)} D_{3}^{(4,2)} \widetilde{S}^{(2)} D_{3}^{(2,4)} \\
& H_{+1}=\widetilde{S}^{(4)} D_{1}^{(4,2)} \widetilde{S}^{(2)} D_{3}^{(2,4)} \\
& H_{-1}=\widetilde{S}^{(4)} D_{3}^{(4,2)} \widetilde{S}^{(2)} D_{1}^{(2,4)} .
\end{aligned}
$$

These terms may all be viewed as paths that connect two points in $\Lambda^{(4)}$. In $H_{0}$ these paths visit $\Lambda^{(2)}$ by either going through $\Lambda^{(1)}$ or $\Lambda^{(3)}$ and then coming back through the same domain. The paths in $H_{ \pm 1}$ visit all four domains, i.e, they wind once around the lattice. In $H_{+1}$ they wind in forward direction, while in $H_{-1}$ they run backwards. According to the above discussion, in all three terms $H_{0}, H_{ \pm 1}$ the paths have a minimum length of $\beta$ steps.

With the help of the definitions in (14) and the trace-log formula for determinants we can now write $W$ as

$$
\begin{aligned}
& W=\operatorname{det}\left[1-H_{0}-e^{\mu \beta} H_{+1}-e^{-\mu \beta} H_{-1}\right] \\
& =\exp \left(-\sum_{n=1}^{\infty} \frac{1}{n} \operatorname{Tr}\left[H_{0}+e^{\mu \beta} H_{+1}+e^{-\mu \beta} H_{-1}\right]^{n}\right) \\
& =\exp \left(-\sum_{q \in \mathbb{Z}} e^{\mu \beta q} \sum_{n=1}^{\infty} \frac{1}{n} \sum_{k_{1}+\ldots+k_{n}=q} \operatorname{Tr}\left[H_{k_{1}} H_{k_{2}} \ldots H_{k_{n}}\right]\right) .
\end{aligned}
$$

In the final step we have rewritten the $n$-th powers by introducing two auxiliary sums: The first sum runs over an integer variable $q$ which below we will identify as the winding number of the individual loop contributions. The second, restricted sum runs over $n$ variables $k_{i} \in\{-1,0,+1\}$.

Due to the trace the individual terms $\operatorname{Tr}\left[H_{k_{1}} H_{k_{2}} \ldots H_{k_{n}}\right]$ correspond to closed loops built from the paths in the $H_{k_{i}}$. Since $H_{+1}$ and $H_{-1}$ contribute a path that winds once in forward (backward) direction and $H_{0}$ does not wind at all, the sum $q=k_{1}+k_{2}+\ldots k_{n}$ is the winding number of the loop. It is obvious from the last expression in (15) that the chemical potential couples to the winding number as expected.

From (15) follows the factorization of $W$ :

$$
W=\prod_{q \in \mathbb{Z}} W^{(q)},
$$

where the factors for the individual winding sectors are given by

$W^{(q)}=\exp \left(-e^{\mu q \beta} \sum_{n=1}^{\infty} \frac{1}{n} \sum_{k_{1}+\ldots+k_{n}=q} \operatorname{Tr}\left[H_{k_{1}} H_{k_{2}} \ldots H_{k_{n}}\right]\right)$.

We remark that there is a relation between the sectors of opposite winding number $q$,

$$
W^{(-q)}(\mu)=W^{(q)}(-\mu)^{*},
$$

where the asterisk denotes complex conjugation. This follows again from the $\gamma_{5}$-hermiticity relations (8) which can be used to establish

$$
\operatorname{Tr}\left[H_{k_{1}} H_{k_{2}} \ldots H_{k_{n}}\right]=\operatorname{Tr}\left[H_{k_{1}^{\prime}} H_{k_{2}^{\prime}} \ldots H_{k_{n}^{\prime}}\right]^{*},
$$

where $k_{1}^{\prime}=-k_{n}, k_{2}^{\prime}=-k_{n-1}, \ldots k_{n}^{\prime}=-k_{1}$. This result immediately leads to (18).

For zero chemical potential the relation (18) reduces to

$$
W^{(-q)}(\mu=0)=W^{(q)}(\mu=0)^{*},
$$

which ensures the well known fact that the determinant is real for vanishing chemical potential $\left(W^{(0)}\right.$ is real anyway). It is quite interesting to note, that due to the relation (20) at $\mu=0$ the contributions from the non-trivial sectors with $q \neq 0$ are positive, since $W^{(-q)}(0) W^{(q)}(0)=\left|W^{(q)}(0)\right|^{2}$. Thus a possible negative sign of the $\mu=0$ determinant must come from the non-winding contributions $A_{0} W^{(0)}$. For $\mu \neq 0$ the relation (20) is violated due to the different weight factors $\exp ( \pm \mu q \beta)$, and the determinant becomes complex.

Let us finally discuss an important aspect of our result: We have noted above that the paths in the terms $H_{k_{i}}$ consist of at least $\beta$ steps. Thus a contribution $\operatorname{Tr}\left[H_{k_{1}} H_{k_{2}} \ldots H_{k_{n}}\right]$ consists of closed loops 


\begin{tabular}{c|ccccccc}
$m$ & $A_{0}$ & $|\operatorname{det}[D]|_{\mu=0}$ & $|W|_{\mu=0}$ & $|\operatorname{det}[D]|_{\mu=0.1}$ & $|W|_{\mu=0.1}$ & $|\operatorname{det}[D]|_{\mu=0.2}$ & $|W|_{\mu=0.2}$ \\
\hline & & & & & & & \\
0.05 & $0.724 \times 10^{47}$ & $0.232 \times 10^{48}$ & 3.202 & $0.223 \times 10^{48}$ & 3.085 & $0.199 \times 10^{48}$ & 2.743 \\
0.10 & $0.179 \times 10^{45}$ & $0.509 \times 10^{45}$ & 2.838 & $0.492 \times 10^{45}$ & 2.740 & $0.440 \times 10^{45}$ & 2.453 \\
0.20 & $0.377 \times 10^{40}$ & $0.875 \times 10^{40}$ & 2.319 & $0.848 \times 10^{40}$ & 2.249 & $0.769 \times 10^{40}$ & 2.039
\end{tabular}

TABLE I: Values of $A_{0},|\operatorname{det}[D]|$ and $|W|$ for three different quark masses, $m=0.05,0.1,0.2$, and three different values of the chemical potential, $\mu=0,0.1,0.2$ for a single configuration.

which have at least a length of $n \beta$. Long loops are suppressed exponentially in $n$, due to the fluctuations of the gauge links and the factors of $\kappa$. Obviously the sum over $n$ corresponds to a generalized hopping expansion.

\section{NUMERICAL ANALYSIS OF THE FACTORIZATION FORMULA}

Various aspects of our determinant factorization can be analyzed numerically. In particular we study the behavior of the factors $A_{0}$ and $W$ as a function of the mass parameter $m$ and the chemical potential $\mu$. For our exploratory analysis we use quenched [10] gauge configurations on lattices of size $8^{3} \times 4$. The configurations were generated with the LüscherWeisz gauge action [6] and at the value of $\beta=7.60$ which we use here, the lattice spacing was determined [7] to be $a=0.193 \mathrm{fm}$. This gives rise to a cutoff of roughly $1 \mathrm{GeV}$ and corresponds to a temperature of $254 \mathrm{MeV}$.

As a first test we programmed both sides of the exact relation (10) in order to test the correct implementation of the terms in (11). We found agreement to machine precision.

In the actual analysis of the properties of the factorization formula we begin with evaluating the factors $A_{0}$ and $W$ (see Eq. (11)) as a function of mass $m$ and chemical potential $\mu$. We refer to $A_{0}$ as the bulk factor, while $W$ is the winding term of the determinant. For the numerical study we consider the mass values $m=0.05,0.1,0.2$, and scan the chemical potential in the interval $\mu \in[0.0,1.0]$ in steps of 0.01 . Concerning the chemical potential we remark, that once the matrices $A_{0}, H_{0}, H_{ \pm 1}$ are evaluated, it is very cheap to compute the $\mu$-dependent winding term $W$ for many values of $\mu$. The reason is obvious from the definition of $W$ in the first line of (15), which shows that the determinant one has to evaluate is only over the indices of a single time slice. This dimensional reduction speeds up the numerical evaluation of the determinant considerably.

To give an idea of the relative size of the different quantities, we show in Table 1 the values of bulk factor $A_{0}$ together with the absolute values $|\operatorname{det}[D]|$ and $|W|$ for three different masses $m=0.05,0.1$ and 0.2 , for a single gauge configuration. Note that $A_{0}$ is independent of $\mu$, while $|\operatorname{det}[D]|$ and $|W|$ do depend on $\mu$, and we thus give the latter two factors for three different values of the chemical potential, $\mu=$ $0.0,0.1,0.2$.

What is immediately obvious from the table, is the rather different scale of the two factors $A_{0}$ and $W$, which according to (10) constitute the determinant, $\operatorname{det}[D]=A_{0} W$. While the bulk factor $A_{0}$ is large of order $\mathcal{O}\left(10^{40}\right)-\mathcal{O}\left(10^{47}\right)$, depending on the mass parameter $m$, the winding term $W$ is of order 1 (at least for the values of $\mu$ in the table). Furthermore $A_{0}$ strongly depends on the mass, while $W$ shows only a weak dependence. We thus conclude that the real valued bulk factor $A_{0}$ contributes the massdependent scale of the determinant, while $W$ brings in the $\mu$-dependence and the complex phase. Thus, besides separating the $\mu$-dependence, our decomposition provides a natural factorization into the relevant scales for the bulk behavior and the response to the chemical potential.

In Fig. 2 we now analyze how the winding term $W$ changes with the chemical potential $\mu$. In the top plot we show the absolute value $|W|$ as a function of $\mu$, while in the bottom plot the phase $\phi$ is displayed. Note that since $A_{0}$ is real, the phase $\phi$ of $W$ is also the phase of $\operatorname{det}[D]$ (up to a possible minus sign that could appear for exceptional configurations). The data in Fig. 2 are for a single configuration which we analyzed for three different values of the quark mass, $m=0.05,0.1,0.2$.

The plots show a non-trivial dependence of $W$ on $\mu$. Starting out with a weak $\mu$-dependence for small $\mu, W$ changes considerably as $\mu$ gets close to the cutoff at $\mu=1$. With increasing $\mu$ the phase $\phi$ runs twice through all of the interval $[-\pi, \pi]$ (note that when exceeding $\pi$ we project the phase back into $[-\pi, \pi])$. What is surprising is that the mass dependence for both, $|W|$ and the phase $\phi$ is rather small. This supports the above sketched picture of $A_{0}$ carrying most of the overall scale and mass dependence.

We remark that we repeated the analysis of Fig. 2 for several configurations and found that the scale aspects of the behavior we describe in the last paragraph are universal: $A_{0}$ carries most of the overall scale of the determinant and also of the mass dependence. The $\mu$-dependence in $W$ shows a much smaller variation. Other aspects are specific for individual configurations, in particular how quickly and 


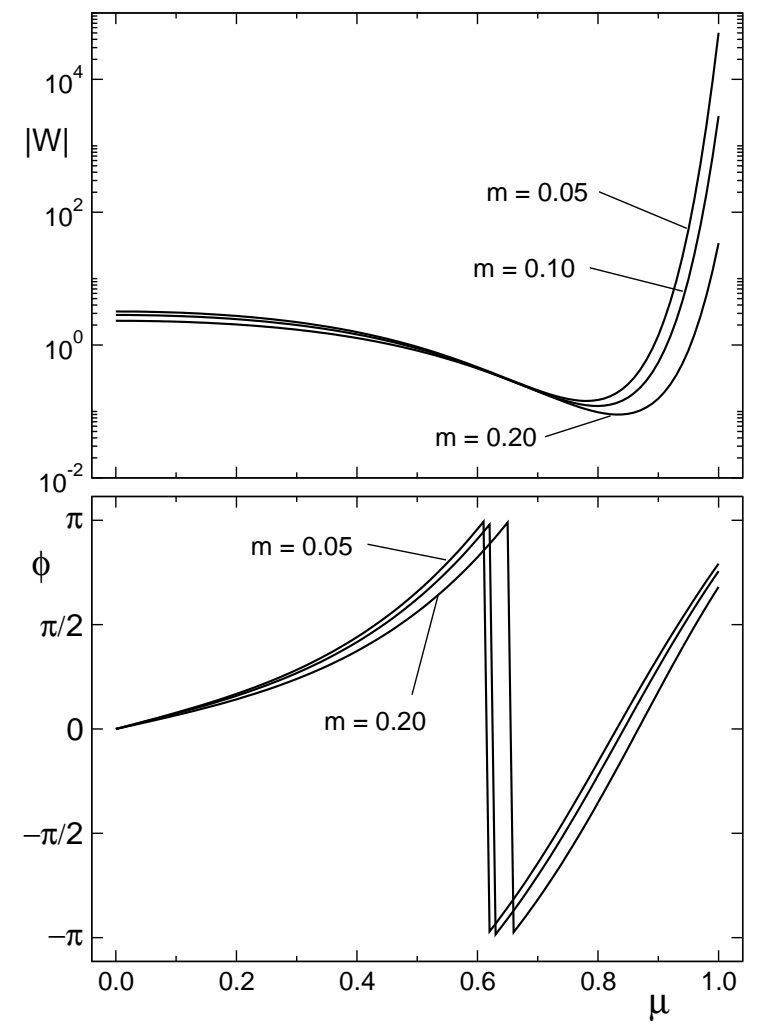

FIG. 2: Absolute value (top plot) and phase (bottom) of the winding term $W$ as a function of $\mu$ for three different masses on a single configuration.

with which orientation the phase $\phi$ is run through as a function of $\mu$. Also the details of the behavior of $|W|$ as a function of $\mu$ may vary for different configurations.

Let us now analyze the buildup of the contributions in the individual winding sectors. For that purpose we write the factor $W^{(q)}$ for winding number $q$ as

$$
W^{(q)}=\exp \left(-e^{\mu q \beta} T^{(q)}\right)
$$

where we define

$$
\begin{aligned}
T^{(q)} & =\sum_{n=1}^{\infty} T_{n}^{(q)}, \\
T_{n}^{(q)} & =\frac{1}{n} \sum_{k_{1}+\ldots+k_{n}=q} \operatorname{Tr}\left[H_{k_{1}} H_{k_{2}} \ldots H_{k_{n}}\right] .
\end{aligned}
$$

It is straightforward to evaluate the coefficients $T_{n}^{(q)}$ and for illustration purposes we list a few terms:

$T_{1}^{(0)}=\operatorname{Tr} H_{0}$,

$T_{2}^{(0)}=\operatorname{Tr} H_{+1} H_{-1}+\frac{1}{2} \operatorname{Tr} H_{0}^{2}$,

$T_{3}^{(0)}=\operatorname{Tr} H_{+1} H_{0} H_{-1}+\operatorname{Tr} H_{+1} H_{-1} H_{0}+\frac{1}{3} \operatorname{Tr} H_{0}^{3}$.

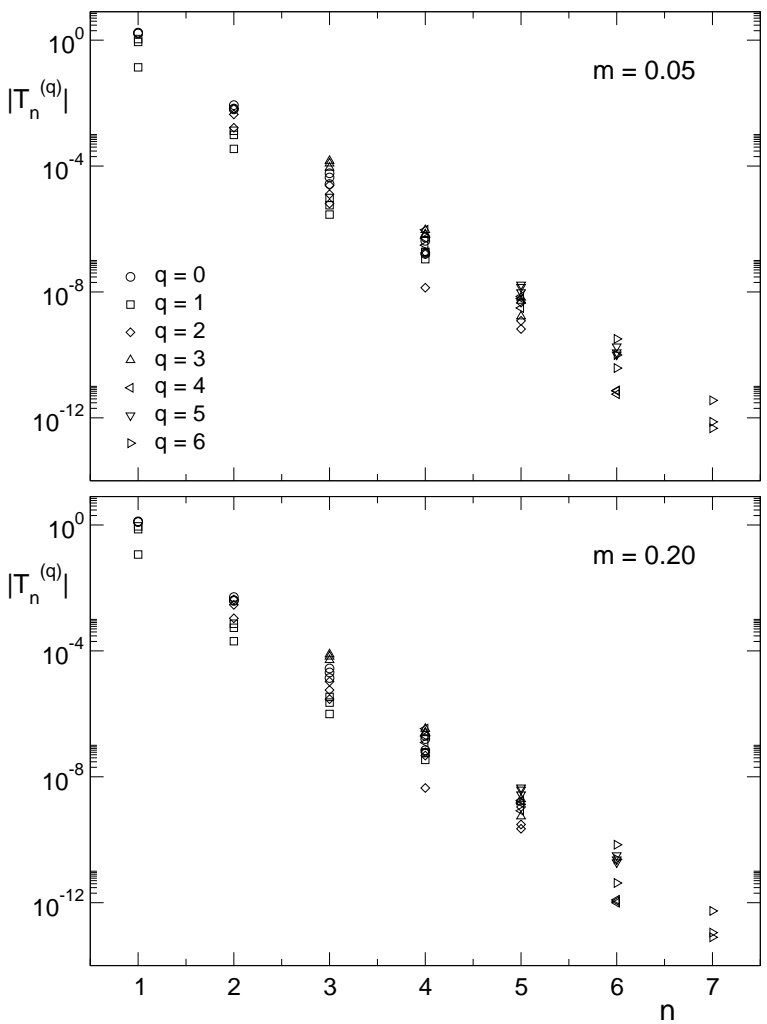

FIG. 3: The absolute values $\left|T_{n}^{(q)}\right|$ for various values of $q$ as a function of $n$. We use two different masses, $m=0.05$ in the plot at the top, and $m=0.20$ at the bottom. We superimpose the data from three different gauge configurations.

$$
\begin{aligned}
& T_{1}^{(1)}=\operatorname{Tr} H_{+1}, \quad T_{2}^{(1)}=\operatorname{Tr} H_{+1} H_{0} \\
& T_{3}^{(1)}=\operatorname{Tr} H_{+1}^{2} H_{-1}+\operatorname{Tr} H_{+1} H_{0}^{2} . \\
& T_{1}^{(2)}=0, T_{2}^{(2)}=\frac{1}{2} \operatorname{Tr} H_{+1}^{2}, T_{3}^{(2)}=\operatorname{Tr} H_{+1}^{2} H_{0} . \\
& T_{1}^{(3)}=0, T_{2}^{(3)}=0, T_{3}^{(3)}=\frac{1}{3} \operatorname{Tr} H_{+1}^{3} .
\end{aligned}
$$

From $q=k_{1}+k_{2}+\ldots k_{n}$ follows $T_{n}^{(q)}=0$ for $q>n$. Note that due to (19) the coefficients obey

$$
T_{n}^{(0)} \in \mathbb{R}, T_{n}^{(-q)}=T_{n}^{(q) *} .
$$

We stress again that the minimal length of the loops in a coefficient $T_{n}^{(q)}$ is $n \beta$, such that we expect the $T_{n}^{(q)}$ to decrease exponentially with $n$.

The evaluation of the $T_{n}^{(q)}$ may numerically be implemented efficiently by pre-calculating often used combinations such a $H_{ \pm 1}^{2}, H_{ \pm 1} H_{0}$ etc. Note that all these terms only live on the single time slice $\Lambda^{(4)}$. Thus the storage requirements are modest and the 
matrix multiplications and traces needed for the $T_{n}^{(q)}$ run only over the indices in a single time slice.

In Fig. 3 we show the absolute values $\left|T_{n}^{(q)}\right|$ for various $q=0,1 \ldots 7$ as functions of $n$. We compare two values of the mass parameter, using $m=0.05$ in the top plot and $m=0.20$ at the bottom. To illustrate the fluctuation of the absolute values we superimpose the data points from three different gauge configurations. The plots nicely display the expected exponential decay with increasing $n$, with the data points for different $q$ and different configurations all being close to a straight line. It is interesting to note, that although between the top and the bottom plots the mass was increased by a factor of 4 , the slope of this line changes only very little. This is not a surprise, since the mass $m$ enters through powers of $\kappa=1 /(4+m)$ which for the range of masses considered in the plots is only a slowly varying function. Also for the phase of the $T_{n}^{(q)}$ we find only a very small mass dependence. The weak mass dependence of the $T_{n}^{(q)}$ then in turn implies the weak mass dependence of $W$ which we have observed in Fig. 2 and Table I.

\section{APPLICATIONS OF THE FACTORIZATION FORMULA}

The winding decomposition formula can be applied to the determination of the canonical partition functions for a fixed quark or baryon number. The grand canonical partition function $Z_{G C}(\mu)$ at chemical potential $\mu$ is obtained by integrating the determinant over the gauge fields,

$$
Z_{G C}(\mu)=\int \mathcal{D}[U] e^{-S_{G}[U]} \operatorname{det}[D(\mu)],
$$

where $S_{G}[U]$ is the gauge action, and for simplicity we discuss the case of only one quark flavor.

The grand canonical partition function may be written as a sum over canonical partition functions $Z^{(Q)}$ with a fixed quark number $Q$,

$$
Z_{G C}(\mu)=\sum_{Q} e^{\mu Q \beta} Z_{C}^{(Q)}=\sum_{B} e^{3 \mu B \beta} Z_{C}^{(3 B)},
$$

where in the second step we have made explicit the fact that due to the center symmetry only the canonical partition functions $Z_{C}^{(3 B)}$, where the quark number is a multiple of 3 , are non-vanishing. $B$ is the baryon number. We remark, that for expectation values also quark sectors where $Q$ is not a multiple of 3 contribute, and furthermore stress that the center symmetry may be broken at the phase transition.

Our winding decomposition formula may now be used to determine the canonical partition functions in various effective ways.

\section{A. Projected determinants from the Fourier transformation method}

The canonical partition functions may, e.g., be determined from a Fourier transformation with respect to an imaginary chemical potential $\mu=i \varphi / \beta$ :

$$
Z_{C}^{(Q)}=\int \mathcal{D}[U] e^{-S_{G}[U]} D^{(Q)},
$$

where we define the projected determinants $D^{(Q)}$,

$$
D^{(Q)}=\int_{-\pi}^{\pi} \frac{d \varphi}{2 \pi} e^{-i Q \varphi} \operatorname{det}[D(\mu=i \varphi / \beta)] .
$$

Although the determination of the $D^{(Q)}$ through Fourier transformation assumes the elegant form of Eq. (31), it is expensive in an actual implementation. The $\varphi$ integral has to be done numerically and experience shows that a large number of $\varphi$-values is needed for an accurate approximation of the $\varphi$ integral. Thus the determinant $\operatorname{det}[D(\mu=i \varphi / \beta)]$ needs to be evaluated many times for different $\varphi$.

With the factorization formula this procedure may be accelerated considerably. Using (10), (15) we find

$$
\begin{aligned}
D^{(Q)}= & \frac{A_{0}}{2 \pi} \int_{-\pi}^{\pi} d \varphi e^{-i Q \varphi} \\
& \times \operatorname{det}\left[1-H_{0}-e^{i \varphi} H_{+1}-e^{-i \varphi} H_{-1}\right] .
\end{aligned}
$$

Also here a determinant is integrated over $\varphi$ and thus has to be evaluated at many values of $\varphi$. However, the determinant in (32) is for a considerably smaller matrix, since $H_{0}, H_{ \pm 1}$ live on only a single timeslice. The strategy thus is to pre-calculate $H_{0}, H_{ \pm 1}$ and $A_{0}$ and to work with (32) instead of (31). The dimension of the problem is reduced by a factor of $\beta$, the number of timeslices. Since the cost for the exact evaluation of a determinant is proportional to the third power of the matrix size, one gains a factor of $\beta^{3}$, which for typical lattice sizes is an improvement of $\mathcal{O}(100)$. The increased efficiency due to the dimensional reduction allows for a considerably larger number of values $\varphi$ used in the numerical integration, such that the accuracy of the results for the projected determinants improves.

\section{B. Projected determinants from a power series expansion}

In addition to the Fourier transformation technique, the equations derived in Section III allow also for an efficient determination of the projected determinants $D^{(Q)}$ via a power series expansion. From (28) - (30) follows the fugacity expansion

$$
\operatorname{det}[D(\mu)]=\sum_{Q} e^{\mu Q \beta} D^{(Q)},
$$


which shows that the $D(Q)$ may be obtained as the coefficients of a series expansion of $\operatorname{det}[D(\mu)]$.

Using the notation of the last two sections we can write the determinant as

$$
\begin{aligned}
\operatorname{det}[D(\mu)] & =A_{0} W^{(0)} \\
& \times \prod_{q=1}^{\infty} \exp \left(-e^{\mu q \beta} T^{(q)}-e^{-\mu q \beta} T^{(q) *}\right) .
\end{aligned}
$$

Expanding the exponential functions and ordering the terms with respect to the powers of $e^{ \pm \mu \beta}$ we can read off the projected determinants $D^{(Q)}$

$$
\begin{aligned}
& D^{(0)}=A_{0} W_{0}\left(1+\sum_{q=1}^{\infty}\left|T^{(q)}\right|^{2}+\frac{1}{4} \sum_{q=1}^{\infty}\left|T^{(q)}\right|^{4} \ldots\right), \\
& D^{(1)}=A_{0} W_{0}\left(-T^{(1)}+\sum_{q=1}^{\infty} T^{(q+1)} T^{(q) *}+\ldots\right), \\
& D^{(2)}=A_{0} W_{0}\left(-T^{(2)}+\sum_{q=1}^{\infty} T^{(q+2)} T^{(q) *}+\ldots\right),
\end{aligned}
$$

et cetera .

Again we can obtain the $D^{(Q)}$ for negative $Q$ via complex conjugation, $D^{(-Q)}=D^{(Q) *}$. Note that a term $T^{(q)}$ consists of loops that wind at least $n=q$ times and thus according to the numerical analysis higher terms in (35) are exponentially suppressed, and the sums may be truncated after a few leading terms. We remark that the expansion which we have denoted here for a single flavor may easily be generated to an arbitrary number of flavors.

\section{A simple truncation scheme}

Having worked out the evaluation of the contributions in the individual winding sectors we now address the question of a suitable truncation scheme. Obviously the $T^{(q)}$ are given by infinite sums (see Eq. (22)) which cannot be summed in closed form. However, the exponential decrease of the individual terms $T_{n}^{(q)}$, which was illustrated in Fig. 3, suggests that a truncation of the sums might lead to a reasonable approximation scheme. We experimented with truncated contributions $\widehat{T}^{(q)}$ defined as

$$
\begin{aligned}
& \widehat{T}^{(0)}=\sum_{n=1}^{1+\Delta} T_{n}^{(0)}, \\
& \widehat{T}^{(q)}=\sum_{n=|q|}^{|q|+\Delta} T_{n}^{(q)} \text { for } q \neq 0 .
\end{aligned}
$$

The parameter $\Delta$, which is a positive integer, controls the approximation and in the limit $\Delta \rightarrow \infty$ the exact result is obtained. Since the $T_{n}^{(q)}$ decay exponentially in $n$, it may be expected that already a

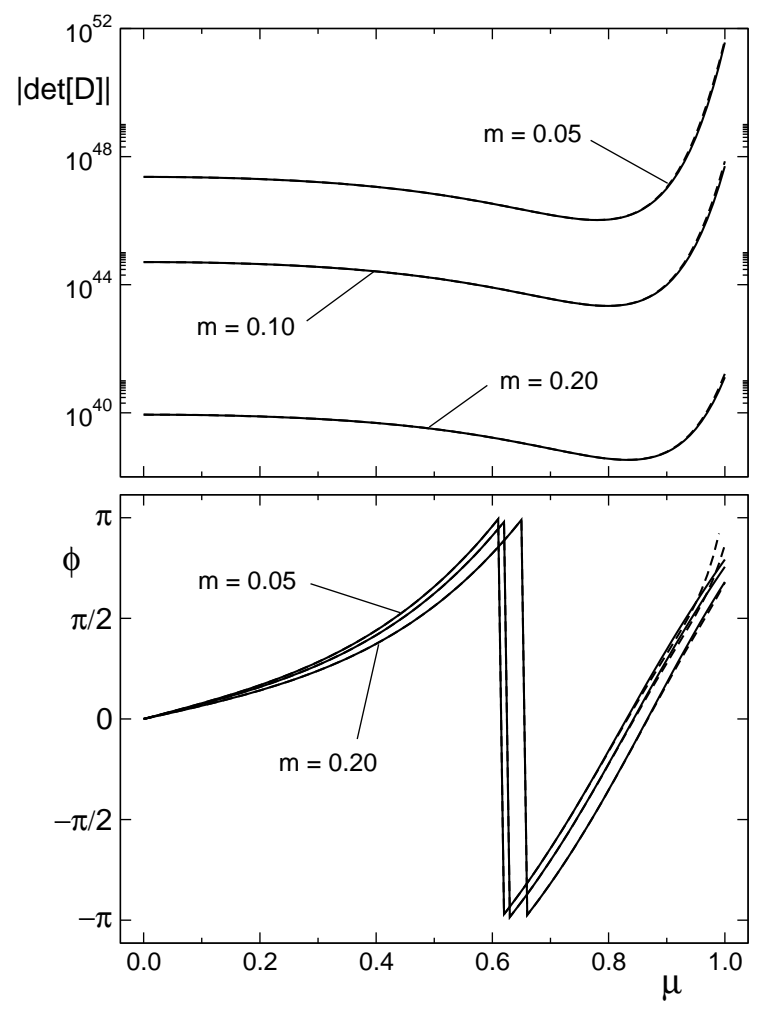

FIG. 4: Absolute value (top plot) and phase $\phi$ (bottom) of the determinant $\operatorname{det}[D]$ as a function of $\mu$. For an individual gauge configuration and three different masses we compare the exact result (full curves) to the approximation discussed in Section VI.C. for $\Delta=1$ (dashed curves).

small value of $\Delta$ might give a good approximation. In fact Fig. 3 shows that for this case subsequent terms are suppressed by a factor of $\mathcal{O}(100)$.

A particularly simple expansion is obtained for $\Delta=1$. In this case one finds

$$
\begin{aligned}
& \widehat{T}^{(0)}=\operatorname{Tr}\left[H_{0}\right]+\frac{1}{2} \operatorname{Tr}\left[\left(H_{0}\right)^{2}\right]+\operatorname{Tr}\left[H_{+1} H_{-1}\right], \\
& \widehat{T}^{(q)}=\frac{1}{q} \operatorname{Tr}\left[\left(H_{+1}\right)^{q}\right]+\operatorname{Tr}\left[\left(H_{+1}\right)^{q} H_{0}\right],(q>0) .
\end{aligned}
$$

Each term (except for $q=0$ ) consists of only two traces. Furthermore the arguments of the traces have a particularly simple form which allows for a efficient recursive implementation of the needed matrix products.

In order to test the quality of the truncation at $\Delta=1$, we evaluated the determinant according to (34), replaced the $T^{(q)}$ by the $\widehat{T}^{(q)}$ as given in (37), and took into account all terms with $q=0,1 \ldots 6$.

In Fig. 4 we compare the outcome of the truncated determinant (dashed curves) to the exact result (full curves). The comparison is done on a single gauge configuration for three different masses. The plot shows that already the $\Delta=1$ approximation works 
surprisingly well. For most of the $\mu$-range the approximation falls exactly on top of the exact curve and only when $\mu$ reaches the cutoff at $\mu=1$, deviations of the approximation (dashed curve) become visible. The onset of these deviations may be pushed to even higher $\mu$ by including larger $q$ in (34), while still sticking to the $\Delta=1$ approximation. For the quenched example studied here this is not necessary, since the deviation occurs only close to the cutoff.

We repeated this analysis for several gauge configurations, and found the same quality of approximation throughout. Thus we conclude that the truncation (36) together with the series expansion of Section VI.B. provides a powerful method for computing the projected determinants $D^{(Q)}$.

\section{CONCLUDING REMARKS}

In this paper we have revisited the problem of finding the fermion determinant in a sector with a fixed quark number, i.e., projecting to a fixed winding number of the closed loops the determinant consists of. In our approach we use a partition of the lattice into four domains and the determinant is expressed in terms of sub-determinants and propagators on the domains. The determinant can be organized in such a way that the winding numbers of the individual contributions can be accessed efficiently.

Our result allows for two different new approaches for computing the projected determinant at a fixed winding number: The factorization of the determinant dimensionally reduces the piece which depends on the chemical potential to a single time slice. Thus the evaluation of the determinant for different values of the imaginary potential $\varphi$ becomes considerably cheaper, and many more $\varphi$-points can be used in the numerical Fourier transformation to the determinant at fixed quark number. A second more direct approach is a power series expansion of the determinant, where the terms at fixed winding number appear as expansion coefficients. We propose a suitable truncation scheme for this expansion which we analyzed numerically.

The method of a partition into domains may be used also for other applications. In particular it exists also for propagators and allows for the analysis of the winding numbers of the paths the propagators consist of. This might be useful for the recently proposed dual chiral condensate [8], where the Fourier transform approach is used to define observables that are sensitive to chiral symmetry breaking as well as confinement. The domain factorization of therm propagator might also be combined with a pseudofermion representation of the fermion determinant and so open another approach to lattice QCD with chemical potential.

Acknowledgments: We thank Falk Bruckmann, Philippe de Forcrand, Christian Lang and Keh-Fei Liu for interesting and helpful discussions. This work is supported by the Austrian FWF doctoral college DK W1203. C.G. thanks the Galileo-Galilei Institute for Theoretical Physics for the hospitality, and the INFN for partial support during the completion of this work.
[1] A. Hasenfratz and D. Toissant, Nucl. Phys. B 371, 539 (1992).

[2] J. Engels, O. Kaczmarek, F. Karsch, and E. Laerman, Nucl. Phys. B 642, 290 (1999).

[3] K.-F. Liu, Int. J. Mod. Phys. B 16, 2017 (2002); K.F. Liu, in: QCD and Numerical Analysis, Vol. III (Springer, New York, 2005), p. 101; A. Alexandru, M. Faber, I. Horvath and K.-F. Liu, Phys. Rev. D 72, 114513 (2005); A. Alexandru, M. Faber, I. Horvath and K.-F. Liu, Nucl. Phys. B, Proc. Suppl. 140, 517 (2005); A. Li, A. Alexandru and K. F. Liu, PoS LAT2006, 030 (2006); PoS LAT2007, 203 (2007); A. Alexandru, A. Li and K. F. Liu, PoS LAT2007, 167 (2007).

[4] S. Kratochvila and P. de Forcrand, Nucl. Phys. Proc. Suppl. 140, 514 (2005); S. Kratochvila and P. de Forcrand, PoS LAT2005, 167 (2006); P. de Forcrand and S. Kratochvila, Nucl. Phys. Proc. Suppl. 153, 62 (2006).

[5] P.E. Gibbs, Phys. Lett. B 172, 53 (1986).

[6] M. Lüscher and P. Weisz, Commun. Math. Phys. 97, 59 (1985), Err.: 98, 433 (1985); G. Curci, P. Menotti and G. Paffuti, Phys. Lett. B 130, 205 (1983), Err.:
135, 516 (1984).

[7] C. Gattringer, R. Hoffmann and S. Schaefer, Phys. Rev. D 65, 094503 (2002).

[8] C. Gattringer, Phys. Rev. Lett. 97, 032003 (2006); F. Synatschke, A. Wipf, C. Wozar, Phys. Rev. D 75, 114003 (2007); F. Bruckmann, C. Gattringer, C. Hagen, Phys. Lett. B 647, 56 (2007); C. Hagen, F. Bruckmann, E. Bilgici and C. Gattringer, PoS LAT2007, 289 (2007); E. Bilgici, F. Bruckmann, C. Gattringer and C. Hagen, Phys. Rev. D 77, 094007 (2008); F. Synatschke, A. Wipf and K. Langfeld, Phys. Rev. D 77, 114018 (2008); E. Bilgici and C. Gattringer, JHEP 0805, 030 (2008).

[9] We remark that our definition of the hopping parameter $\kappa$ differs from another often used convention by a factor of 2 , but has the advantage of keeping intact the projectors $\left(1 \pm \gamma_{\mu}\right) / 2$.

[10] It has to be remarked, that the quenched ensemble used here only serves to test properties of the analytical results obtained in this article. Configurations from a fully dynamical ensemble would certainly give quantitatively different results. 